Running head: DAILY REASONS FOR LIVING AND SUICIDAL IDEATION

\title{
Protective Effects of Reasons for Living Against Suicidal Ideation in Daily Life
}

Aliona Tsypes ${ }^{1}$, Aleksandra Kaurin ${ }^{2}$, Aidan G.C. Wright ${ }^{3}$, Michael N. Hallquist ${ }^{4} \&$ Alexandre Y. Dombrovski $^{1}$

\author{
${ }^{1}$ Department of Psychiatry, University of Pittsburgh \\ ${ }^{2}$ Faculty of Health/School of Psychology and Psychiatry, Witten/Herdecke University \\ ${ }^{3}$ Department of Psychology, University of Pittsburgh \\ ${ }^{4}$ Department of Psychology and Neuroscience, University of North Carolina at Chapel Hill
}

Published in the Journal of Psychiatric Research: 10.1016/j.jpsychires.2022.01.060

Acknowledgements: This research was supported by grants from the National Institute of Mental Health (R01MH048463, R01MH100095, R01MH119399, T32MH018269), the University of Pittsburgh's Clinical and Translational Science Institute, which is funded by the National Institutes of Health (NIH) Clinical and Translational Science Award (CTSA) program (UL1TR001857). The CTSA program is led by the NIH's National Center for Advancing Translational Sciences. The opinions expressed are solely those of the authors and not those of the funding source. We would like to thank Timothy A. Allen for his valuable input regarding the visual depiction of our cross-level moderation effects.

Declaration of interest: None.

Correspondence concerning this article should be addressed to Aliona Tsypes, PhD, Department of Psychiatry, University of Pittsburgh, 3811 O’Hara St., Pittsburgh, PA 15213. E-mail: tsypesa@upmc.edu 
How do individuals resist suicidal urges in a crisis? Deterrents for suicide can be conceptualized as reasons for living (RFL), but our understanding of their protective effects is predominantly informed by cross-sectional research. We examined the protective effects of RFL on suicidal ideation (SI) in daily life in a high-risk sample. We also tested whether personality traits moderated the strength of the dynamic RFL-SI link. Adults with a borderline personality disorder diagnosis $\left(N=153, n_{\text {suicide attempters }}=105\right)$ completed a 21 -day ambulatory assessment protocol. Daily endorsements of RFL were negatively linked to SI at the within-person but not the between-person level. Whereas suicide attempters endorsed RFL less frequently than nonattempters, the protective effect of RFL was undiminished in this group. Furthermore, RFL's protective effect was particularly pronounced in those with higher average levels of suicidal ideation. While people high on extraversion endorsed RFL more often, this increase was not protective against SI, indicating that RFL reflect heterogeneous underlying psychological processes, only some of which protect against SI.

Keywords: suicide, borderline personality disorder, reasons for living, ecological momentary assessment, multilevel structural equation modelling 


\section{Protective Effects of Reasons for Living Against Suicidal Ideation in Daily Life}

Suicidal crises emerge and dissipate dynamically over relatively brief periods of time (Kleiman et al., 2017). Whereas the field has accumulated considerable knowledge about the factors that increase the likelihood of suicidal behavior in a relatively distant future (Franklin et al., 2017), little is known about the short-term dynamics of protective factors that reduce suicidal urges. During the state of suicidal ambivalence, characterized by conflicting desires to live and to die, beliefs regarding one's positive expectancies about life may decrease the likelihood and intensity of suicidal thinking. The present study focused on the effects of daily fluctuations in these expectancies (i.e., reasons for living; RFL) on suicidal ideation and examined the relevance of individual differences in personality to these processes.

People who think about suicide tend to engage in an internal debate about their reasons for dying and living (Harris et al., 2019; Shneidman and Farberow, 1957). The interplay of these conflicting motivations has been shown to shape the degree of suicide intent severity, with greater orientation towards death being associated with more serious suicidal intent and vice versa (Brown et al., 2005; Kovacs and Beck, 1977; O'Connor et al., 2012). One intervention towards increasing the motivation to live in individuals with suicidal ideation is to invite reflection on personal RFL, such as those assessed by the Reasons for Living Inventory (RFLI; Linehan et al., 1983). The inventory encompasses a broad range of life-maintaining beliefs and expectations hypothesized to be indicative of deterrents that individuals consider in their struggle to avoid engaging in suicidal behavior. Indeed, a systematic review of the literature suggested that people who struggle with suicidal thoughts report that envisioning RFL helps them resist suicidal ideation (Bakhiyi et al., 2016). 
However, two important unanswered questions remain about the protective effects of RFL. First, since the majority of extant studies were cross-sectional, they provide little information about whether endorsements of RFL are useful for assessing short-term (e.g. at vulnerable times in daily life) changes in risk or are simply associated with low long-term suicide risk. This question is relevant for clinical safety planning and interventions with individuals at risk. Previous work has illustrated that suicidal ideation tends to covary with well-established risk (Kleiman and Nock, 2018) and protective factors for suicide (Coppersmith et al., 2019). Therefore, it is plausible to assume that RFL are partly state-dependent, fluctuating over similarly brief periods of time due to objective (e.g., life stressors) and/or subjective (e.g., depressive symptoms) factors. In line with this assumption, recent preliminary evidence suggests that therapeutic interventions aimed at reducing suicidal behavior likely work by strengthening the wish to live, rather than by weakening the wish to die (Bryan et al., 2016). Thus, due to the dynamic, state-dependent nature of both suicide risk and the consideration of one's RFL, intensive longitudinal designs are needed to capture their interconnections in people's daily lives.

Second, we have limited knowledge about individual differences affecting the dynamics and protective effects of RFL in people's daily lives. In other words, it is yet to be empirically tested whether reflecting on one's RFL during a suicidal crisis is equally protective for all individuals or whether certain individual differences modulate these effects. Personality traits describe relatively stable response tendencies to a broad range of internal and external stimuli and the likelihood of experiencing particular affective and motivational states in a given situation. Therefore, personality traits may affect the likelihood of considering suicide, reflecting on one's RFL, as well as protective effects of RFL against suicidal ideation. Indeed, there is cross-sectional and retrospective evidence that both internalizing and externalizing Big Five 
personality traits increase risk for suicidal ideation and behavior (Szücs et al., 2020, 2018).

Specifically, suicidal ideation, suicide attempts and death by suicide have been most consistently linked with high levels of neuroticism and low levels of extraversion (e.g., Brezo et al., 2006; Duberstein et al., 2000; Heisel et al., 2016; Segal et al., 2012; Useda et al., 2004), with some although mixed - empirical support for the effects of openness to experience (e.g., Duberstein et al., 1994, 2000; Heisel et al., 2016; Tsoh et al., 2005 versus Segal et al., 2012; Useda et al., 2007), agreeableness (Segal et al., 2012), and conscientiousness (Segal et al., 2012). Taken together, whereas cross-sectional and retrospective studies link the Big Five with suicide risk and the endorsement of RFL (Segal et al., 2012), it remains unclear how specific personality traits affect suicide risk and contemplation of one's RFL in daily life and whether the link between these personality traits and suicide risk is explained by RFL.

This study aimed to characterize the daily dynamics of RFL contemplation and their protective effect on suicidal ideation in people with and without a history of attempted suicide. To understand the determintants of this presumably protective link, we further examined how the Big Five would impact daily endorsements of RFL as well as their dynamic link with suicidal ideation. To do so, we collected smartphone-based daily diary ratings over the course of 21 days in individuals diagnosed with borderline personality disorder (BPD; see Kaurin et al., in press, for more details on the sampling procedure). We aimed to sample individuals at high risk for suicide. While chronic suicidal ideation and recurrent suicide attempts are generally common in BPD (Black et al., 2004; Temes et al., 2019), our sample was enriched for a history of medically serious suicide attempts. This design feature helps to isolate the characteristics of prior suicide attempts, which likely increase the seriousness of suicidal behavior (Soloff et al., 2005). Although suicide attempts are not synonymous to death by suicide, individuals with a history of 
high-lethality suicide attempts share characteristics with those who died by suicide, and the lethality of suicide attempts often progresses with repeated attempts (Malone et al., 1995). Measures of RFL were initially developed to examine the mechanisms underlying treatment process and outcome in chronically suicidal individuals and/or individuals with BPD (Linehan et al., 1993). RFL is based on a cognitive-behavioral view of suicidal behavior, assuming that one of the factors differentiating suicidal from nonsuicidal persons (or states) is the content of an individual's belief system. Indeed, suicidal thoughts and behaviors in BPD are negatively linked to the quantity and quality of RFL in these individuals (or reasons to stay alive; Linehan et al., 2015). Thus, consistent with prior cross-sectional evidence (Bakhiyi et al., 2016; Linehan et al., 2015), we anticipated that greater endorsement of RFL would be negatively associated with suicidal ideation at both the within-person (i.e., daily levels of endorsing RFL and suicidal ideation) and between-person (i.e., average levels of endorsing RFL and suicidal ideation over the course of the study) levels. Prior research has not examined the interconnections among the Big Five, RFL, and suicidal ideation. Thus, our analyses connecting these constructs were exploratory.

\section{Method}

Sample. Participants were 153 individuals diagnosed with BPD ( $M_{\text {age: }} 33.62, S D=9.60$; $81 \%$ female). Of these 153 individuals with BPD, $n=105$ had a history of suicide attempts (BPDATT group) and $n=48$ reported no past suicide attempts (BPD-NON group). Among all participants, 79\% identified as White, 1.3\% Asian, 9.1\% Black or African American, 6.5\% as Hispanic and the rest identified as more than one or another race. More than $13.5 \%$ had a yearly income of $\$ 70,000$ and above, $\sim 28 \% \$ 10,000$ - $\$ 14,999.99$ or less, and the remaining 
participants fell in-between these two income brackets. Twenty-seven participants did not provide any income information.

All study procedures were approved by the Institutional Review Board of the University of Pittsburgh (STUDY19050210). Written informed consent was obtained prior to study participation. Participants were drawn from a longitudinal study (Soloff and Chiappetta, 2017), which recruited from inpatient and outpatient clinics and the Greater Pittsburgh community by advertisement, requiring individuals to be between 18 and 45 years of age at the time of enrollment, and excluding individuals with a lifetime diagnosis of any psychotic or bipolar disorder, clinical evidence of organic brain disease, physical disorders or treatments with known psychiatric consequence, and IQ $<70$ measured by the Wechsler Test of Adult Reading (WTAR). DSM-IV/5 defined BPD diagnoses were ascertained with the ICD-10 based International Personality Disorder Examination (IPDE; Loranger, 1999). The BPD-NON group had no lifetime history of suicide attempts. The BPD-ATT group had a history of self-injurious acts with the intent to die within a 1-month period prior to completing the study assessments or had a history of a past suicide attempt with strong current suicidal ideation at the time of study enrollment. Suicide attempt history was verified by a psychiatrist using all available information: participant's report, medical records, and collateral information from the treatment team, family, and friends. Significant discrepancies between these sources led to exclusion from the study.

Baseline Personality Assessments. The Big Five traits were assessed using the 120-item International Personality Item Pool representation of the NEO-PI-R (IPIP-NEO-120; Johnson, 2014). Participants rated the extent to which a characteristic applied to them (e.g., "I make friends easily"), and items were rated on a Likert scale from "very inaccurate" (1) to "very accurate" (5). Reliability for the trait scales was high (mean $\omega=.86$ ). 
Daily Assessments. Participants completed a 21-day ecological momentary assessment protocol within pre-defined time windows, using the MetricWire smartphone application Version 4.2.8 (2019). They received six random surveys per day assessing suicidal ideation during an approximately twelve-hour time window that corresponded to the participants' typical waking hours, along with end of day questions that assessed RFL. Blocked random intervals had a minimum of 90 minutes between surveys and participants were given 20 minutes to initiate a response to each one. Additionally, participants were prompted to complete a second daily survey around their typical bed time and were given three hours to respond. $77.12 \%$ of our participants responded on all 21 days, and $92.81 \%$ responded to at least 14 days worth of surveys. This resulted in 2993 total days, with an average number of 19.56 days per participant. The predictors in this study were assessed at the daily level, whereas suicidal ideation was assessed at the momentary level. Therefore, we created a daily mean score of suicidal ideation to match the response format of both measures for our analyses.

Reasons for Living. We used six items to assess daily RFL, derived from the survival and coping beliefs as well as responsibility to family subscales of the RFLI (Linehan et al., 1983). There is empirical evidence of a negative link between suicidal ideation and both survival and coping beliefs (e.g., Chang et al., 2014; Lee and Oh, 2012; Oquendo et al., 2005; Pinto et al., 1998; Richardson-Vejlgaard et al., 2009) and responsibility to family (e.g., Lee and Oh, 2012; Pinto et al., 1998; Richardson-Vejlgaard et al., 2009) subscales. Importantly for our ecological momentary assessment study design, we chose items expected to vary the most across days, while also capturing concrete positive deterrents, as opposed to items from other RFLI (Linehan et al., 1983) subscales (i.e., child-related concerns, fear of suicide, fear of social disapproval, or moral objections), which were not used in this study. 
Participants were asked to rate on a five point scale ( 1 = "very slightly/not at all" to 5="a great deal") to what extent "Over the last $24 \mathrm{hrs"} \mathrm{they} \mathrm{thought} \mathrm{a)} \mathrm{that} \mathrm{they} \mathrm{can} \mathrm{learn} \mathrm{to} \mathrm{adjust} \mathrm{or}$ cope with their problems; b) that they have control over their life and destiny; c) about their responsibilities to their family; d) about how much they love and enjoy their family; e) about future plans they look forward to carrying out; f) that everything has a way of working out for the best. To be consistent with the scoring of the original Reasons for Living Inventory (Linehan et al., 1983), we used the composite score of all daily RFL items (McDonald's $\omega_{\text {within }}=.79$; $\omega_{\text {between }}=.92$ ).

Suicidal Ideation. Suicidal ideation was assessed with two dichotomous items $(1=$ yes, 0 = no) from the Columbia Suicide Severity Rating Scale (Posner et al., 2011): "Have you wished you were dead or wished you could go to sleep and not wake up?", "Have you actually had any thoughts of killing yourself?" (McDonald's $\left.\omega_{\text {within }}=.82 ; \omega_{\text {between }}=.92\right)$.

Negative Affect. Participants rated the degree to which they experienced negative affect (i.e., angry, nervous, sad, guilty, ashamed, irritated; McDonald's $\omega_{\text {within }}=.83 ; \omega_{\text {between }}=.93$ ). The items were derived from the Positive and Negative Affect Schedule (Watson et al., 1988) and read "How [ADJECTIVE] do you feel right now?" Ratings were made on a scale from 1 ("Not at All”) to 5 (“A great deal").

Data Analysis. Repeatedly sampling protective factors of suicide results in a hierarchical data structure: Daily RFL assessments (within-person level) are nested within individuals (between-person level). Partitioning the variance into each level provides information about the proportion of variance attributable to individual differences in RFL ratings and to to withinperson daily fluctuations in these ratings. We calculated the intraclass correlation (ICC), which 
can be interpreted as the proportion of total variance accounted for at the between-person level. Consequently, 1.0 - ICC captures the proportion of within-person variance.

For the second portion of our analyses, we employed multilevel structural equation modeling (MSEM). This framework allowed us to test whether daily departures from one's mean-level of RFL endorsements tended to co-occur with deviations in one's subjectively reported suicidal ideation, and whether average levels of RFL and suicidal ideation as well as their respective within-person association were affected by individual differences in the Big Five personality traits. MSEM can accommodate the nested data structure by partitioning the variability in daily ratings into between- and within-person variance. At each level, MSEM can be used to examine associations among variables (Sadikaj et al., 2021). The between-person portion of the model estimates associations among individual differences in each observed variable, akin to coefficients derived from cross-sectional designs such as the correlation or regression path between how suicidal a person tends to be with how strongly they typically tend to experience RFL.

In contrast, within-person associations reflect how strongly variables are dynamically coupled together as they fluctuate from day to day, offering a quantification as to whether RFL might serve as a target for risk monitoring and therapy. MSEM further allows estimation of random slopes (i.e., slopes that vary across individuals). The variability in those random slopes can be understood as another between-person variable that may be related to additional betweenperson variables, assessed outside of the ambulatory assessment protocol. All models were estimated in Mplus with Bayesian parameter estimation (version 8; Muthén \& Muthén, 19982017). Missing data were assumed to be missing at random, and accommodated using a Bayesian approach that uses all available data in estimation. With increasingly large samples, it provides 
similar results to Full Information Maximum Likelihood to address missing data (Asparouhov \& Muthen, 2010).

To evaluate whether the overall severity of suicidal ideation as well as endorsements of RFL differed across our study groups, we created dummy-coded variables indicating whether a participant belonged to the BPD-NON or BPD-ATT group. We then regressed daily suicidal ideation on RFL ratings at the within-person level, and the same structure was mirrored at the between-person level. To examine the temporal precedence of the relation between daily RFL and daily suicidal ideation, we also conducted two lagged analyses examining a) the effects of same-day RFL on next-day suicidal ideation, adjusting for same-day suicidal ideation; b) the effects of same-day suicidal ideation on next-day RFL, adjusting for same-day RFL. In addition, to rule out alternative explanations, we adjusted for daily levels of negative affect in a sensitivity analysis. In a second step, we introduced baseline measurements of the Big Five personality traits as moderators of the same-day within-person path (i.e., cross-level interaction), as well as predictors of individual differences in RFL and suicidal ideation. With regard to the cross-level interactions, regression coefficients denote the extent to which the association of two daily diary variables depends on an individual difference variable (e.g., extraversion).

Significance for all model parameters was based on 95\% Credibility Intervals (CIs), with CIs that excluded zero being indicative of a parameter that differed significantly from zero. Sex $(0=$ female; $1=$ male $)$ and age (centered on mean age $)$ were included as covariates in all models at the between-person level, and day number (i.e., day centered on mean of observations) as well as weekday vs. weekend were entered as within-person covariates. Along with other parameters not reported in the tables (e.g., residual variances), coefficients for covariates are not depicted in 
the diagrams, but full specifications and detailed output from all models can be found online at https://osf.io/tfz43/.

\section{Results}

Table 1 details descriptive values for the total sample and across study groups for the key variables in our study. As expected, levels of suicidal ideation were lower in the BPD-NON group compared to the BPD-ATT group $(\beta=.16, \mathrm{CI}: .01 ; .29)$. Daily endorsements of RFL were lower in the BPD-ATT group ( $\beta=-.15$, CI: -.31; -.01) compared to the BPD-NON group (see https://osf.io/tfz43/ for detailed output files of between-group differences).

Stability and Variability of RFL in Daily Life. We first examined what proportion of variance in daily RFL experiences was attributable to between-person differences. The mean ICC was $.52($ range $=.49-.63)$ at item level and .63 for total score. This suggests that, on average, approximately half of the variance in the daily RFL experiences can be attributed to stable individual differences and the remaining portion can be attributed to daily fluctuations. Although all RFL items were overall comparable with regard to their ICCs, the items assessing plans for the future and coping beliefs had the lowest ICCs, whereas assessments of family love were associated with the largest ICCs (see Table 1 for details).

Daily Associations among RFL and suicidal ideation. Next, we tested the hypothesis that higher daily levels of RFL endorsements would be negatively linked with suicidal ideation both within- and between-persons (Table 2). We found a significant negative link within-person, but not between-persons, suggesting that on days when participants endorsed more RFL they also tended to report lower levels of suicidal ideation. The magnitude of this effect was similar across the BPD-NON and BPD-ATT groups and most pronounced-i.e., more strongly negative - among those with higher average levels of suicidal ideation. A person's tendency to 
experience RFL on average, however, was not related to average levels of suicidal ideation. Further, in the lagged analysis, same-day RFL predicted next day suicidal ideation at the withinperson level, adjusting for same-day suicidal ideation ( $\beta=-.05$, CI: -.09;-01). In contrast, in a separate analysis, the within-person effect of same-day suicidal ideation on next-day RFL was not significant, adjusting for same-day RFL ( $\beta=-.002$, CI: -.09;05). Finally, to substantiate our finding and rule out the plausible alternative that the protective effect of RFL might unfold via affect-regulatory pathways, we controlled for daily levels of negative affect in a sensitivity analysis, but this did not change the main finding. Respective output files can be found under https://osf.io/tfz43/.

Associations with and Moderation by Personality. In a final set of analyses, we examined the links among individual differences in personality traits, RFL, and suicidal ideation. Specifically, we tested whether within-person associations between RFL and suicidal ideation were moderated by individual differences in personality traits (i.e., cross-level interaction). Coefficients for the "trait $\rightarrow$ suicidal ideation," "trait $\rightarrow$ RFL," and "trait $\rightarrow$ random slope" paths of our models are reported in Table 3 . We found that greater openness to experience ( $\beta=.20, \mathrm{CI}$ : $.04 ; .40)$ and extraversion $(\beta=.36$, CI: $.19 ; .52)$ and lower neuroticism $(\beta=-.29$, CI: $-.44 ;-.12)$ were associated with higher average endorsements of RFL. Finally, we found that at high levels of extraversion ( $\beta=.28$, CI: .06; .44), the negative link between RFL and suicidal ideation was dampened. More specifically, this link was only significant at the mean ( $\beta=-.18$, CI: $-.29 ;-.08)$ and one standard deviation (SD) below the mean $(\beta=-.34, \mathrm{CI}:-.48 ;-.21)$ of extraversion. An illustration of this significant interaction effect is provided in Figure 1. In contrast, neuroticism, conscientiousness, openness to experience, and agreeableness did not significantly moderate this link. One plausible alternative explanation is that this pattern represents a ceiling effect (i.e., 
restricted variance), wherein lower levels of extraversion are more and higher levels less variable in RFL. To test this possibility, we ran a sensitivity analysis, correlating the random within-person residuals with extraversion. This model, however, suggested that higher levels of extraversion were linked to more variability in RFL, providing evidence against this alternative explanation.

\section{Discussion}

The present study examined endorsements of RFL and their association with suicidal ideation in daily life along with the moderating effects of the Big Five personality traits on this dynamic link in individuals with BPD with and without a history of attempted suicide. Daily endorsements of RFL were negatively linked to levels of suicidal ideation at the within- but not between-person level. Thus, on days when individuals with BPD thought more about their RFL they were also less likely to think of suicide, but individuals with more RFL on average did not necessarily report lower levels of suicidal ideation. Further, these daily fluctuations accounted for about $40 \%$ of the overall variance in the experiences of RFL, while $60 \%$ reflected an individual's average level, suggesting somewhat more trait-like than state-like variance in RFL. Notably, although suicide attempters endorsed RFL less frequently than non-attempters, the magnitude of the protective within-person effect of RFL was similar across these two groups and was particularly pronounced in those with higher average levels of suicidal ideation. In addition, although caution should be used when considering causality in an observational study, our lagged analyses provide initial evidence that greater same-day RFL predicted lower next-day suicidal ideation, whereas same-day suicidal ideation did not predict next-day RFL.

With regard to personality traits, individuals high on extraversion endorsed thinking of RFL on more days, which builds on and extends prior cross-sectional evidence of the positive association between extraversion and RFL (Segal et al., 2012). This is consistent with the work 
suggesting that extraversion is reflective of behavioral exploration and engagement with rewards (DeYoung, 2015). The current findings extend previous research by also showing that extraversion weakened the dynamic protective within-person effect of RFL against daily suicidal ideation. This effect was particularly enhanced among those with average or low levels of extraversion (see Figure 1). This suggests that the effects of extraversion on RFL are not antisuicidal and likely reflect the psychological processes that are distinct from those involved in the antisuicidal effects of RFL. Thus, the experience of RFL is reflective of heterogeneous cognitive and affective processes, only some of which are protective against suicidal ideation.

The initial evidence of dynamic (and state-dependent) qualities of daily endorsements of RFL and the protective effects of these endorsements on the levels of daily suicidal ideation has implications for suicide risk assessment and intervention. Whereas numerous psychotherapies and interventions aimed at reducing suicide risk focus on the identification and enhancement of RFL either directly (e.g., Dialectical Behavior Therapy, Collaborative Assessment and Management of Suicidality) or indirectly (e.g., Acceptance and Commitment Therapy, Mindfulness-Based Cognitive Therapy), closer focus on the accessibility of daily RFL and their fluctuations might be particularly important for suicide prevention. Indeed, although suicide attempters had fewer RFL than non-attempters, the protective effects of RFL against suicidal ideation were comparable across these two groups. This suggests that interventions that render RFL more salient in the moment can prevent a suicidal crisis. The findings also suggest that these interventions might be particularly fruitful for individuals with elevated average levels of suicidal ideation. Further, it might be beneficial to focus on activating and increasing the positive emotional value of contemplating RFL inside and outside of therapy sessions, for example, through savoring the moment (Klibert et al., 2019) or positive affect stimulation (McMakin et al., 
2011). In addition to highlighting a broad range of RFL, helping patients to come up with ways of recalling their RFL, along with the positive emotional effects linked with these RFL, might serve as an additional componenent of crisis intervention when an individual is at a particularly high risk of engaging in suicidal behavior.

The present study had several limitations that provide directions for future research. First, our study sample was relatively homogenous — predominantly Caucasian females who had been diagnosed with BPD. Future studies in more diverse groups can examine the generalizability of our findings. Second, we assessed RFL at the daily level and thus also created daily average scores of momentary assessments of suicidal ideation over each assessment day. Although aligned with previous work (Coppersmith et al., 2019; Crowe et al., 2019; Kleiman et al., 2017; Restifo et al., 2015; Witte et al., 2006), this approach precluded capturing within-day cofluctuations of RFL and suicidal ideation. Third, to minimize participant burden, we were only able to include six items assessing RFL in the daily diary part of our study. Because these items focused on the domains of survival and coping beliefs (i.e., confidence in one's ability to adequately handle life; positive expectations about the future) and responsibility to/enjoyment of one's family, future work should examine whether our findings generalize to other previously identified domains, such as fear of suicide, moral objections, fear of social disapproval, childrelated concerns (Linehan et al., 1983). Further, to obtain a more idiosyncratic understanding of the protective effects of considering deterrents in daily life, future intensive longitudinal studies could inquire about the RFL that participants themselves select as most important. Finally, this study could not directly elucidate the role of RFL in suicidal behavior, although the indirect inference from contrasting attempters with non-attempters is that the impact of RFL on suicide risk is more likely mediated by suicidal ideation. Future work should elucidate the mechanisms 
underlying the effects of RFL on suicide risk and generalizability of these mechanisms to the state of suicidal crisis.

In conclusion, the present study provides initial evidence of the dynamic link between RFL and suicidal ideation in daily life in high-risk for suicide individuals with BPD. This link was dampened by the Big Five personality trait of extraversion, suggesting that contemplation of one's RFL involves a range of cognitive and affective processes not all of which are protective against suicidal thinking. Finally, although people who had attempted suicide reported fewer RFL, the protective effect of daily RFL appeared intact, suggesting opportunities for intervention. 


\section{Acknowledgements}

This research was supported by grants from the National Institute of Mental Health (R01MH048463, R01MH100095, R01MH119399, T32MH018269), the University of Pittsburgh's Clinical and Translational Science Institute, which is funded by the National Institutes of Health (NIH) Clinical and Translational Science Award (CTSA) program (UL1TR001857). The CTSA program is led by the NIH's National Center for Advancing Translational Sciences. The opinions expressed are solely those of the authors and not those of the funding source. We would like to thank Timothy A. Allen for his valuable input regarding the visual depiction of our cross-level moderation effects. 


\section{References}

Asparouhov, T. and Muthén, B. (2010) Bayesian Analysis Using Mplus: Technical Implementation. Mplus Technical Report. http: \|www.statmodel.com

Bakhiyi, C.L., Calati, R., Guillaume, S., Courtet, P., 2016. Do reasons for living protect against suicidal thoughts and behaviors? A systematic review of the literature. J. Psychiatr. Res. 77, 92-108. https://doi.org/10.1016/j.jpsychires.2016.02.019

Black, D.W., Blum, N., Pfohl, B., Hale, N., 2004. Suicidal behavior in borderline personality disorder: prevalence, risk factors, prediction, and prevention. J. Personal. Disord. 18, 226-239. https://doi.org/10.1521/pedi.18.3.226.35445

Brezo, J., Paris, J., Turecki, G., 2006. Personality traits as correlates of suicidal ideation, suicide attempts, and suicide completions: a systematic review. Acta Psychiatr. Scand. 113, 180 206. https://doi.org/10.1111/j.1600-0447.2005.00702.x

Brown, G.K., Steer, R.A., Henriques, G.R., Beck, A.T., 2005. The internal struggle between the wish to die and the wish to live: A risk factor for suicide. Am. J. Psychiatry 162, 19771979. https://doi.org/10.1176/appi.ajp.162.10.1977

Bryan, C.J., Rudd, M.D., Peterson, A.L., Young-McCaughan, S., Wertenberger, E.G., 2016. The ebb and flow of the wish to live and the wish to die among suicidal military personnel. J. Affect. Disord. 202, 58-66. https://doi.org/10.1016/j.jad.2016.05.049

Chang, W.C., Chen, E.S.M., Hui, C.L.M., Chan, S.K.W., Lee, E.H.M., Chen, E.Y.H., 2014. The relationships of suicidal ideation with symptoms, neurocognitive function, and psychological factors in patients with first-episode psychosis. Schizophr. Res. 157, $12-$ 18. https://doi.org/10.1016/j.schres.2014.06.009

Coppersmith, D.D.L., Kleiman, E.M., Glenn, C.R., Millner, A.J., Nock, M.K., 2019. The dynamics of social support among suicide attempters: A smartphone-based daily diary study. Behav. Res. Ther. 120, 103348. https://doi.org/10.1016/j.brat.2018.11.016

Crowe, E., Daly, M., Delaney, L., Carroll, S., Malone, K.M., 2019. The intra-day dynamics of affect, self-esteem, tiredness, and suicidality in Major Depression. Psychiatry Res. 279, 98-108. https://doi.org/10.1016/j.psychres.2018.02.032

DeYoung, C.G., 2015. Cybernetic Big Five Theory. J. Res. Personal., Integrative Theories of Personality 56, 33-58. https://doi.org/10.1016/j.jrp.2014.07.004

DeYoung, C.G., 2006. Higher-order factors of the Big Five in a multi-informant sample. J. Pers. Soc. Psychol. 91, 1138-1151. https://doi.org/10.1037/0022-3514.91.6.1138

DeYoung, C.G., Peterson, J.B., Higgins, D.M., 2002. Higher-order factors of the Big Five predict conformity: Are there neuroses of health? Personal. Individ. Differ. 33, 533-552. https://doi.org/10.1016/S0191-8869(01)00171-4

Digman, J.M., 1997. Higher-order factors of the Big Five. J. Pers. Soc. Psychol. 73, 1246-1256. https://doi.org/10.1037//0022-3514.73.6.1246

Duberstein, P.R., Conwell, Y., Caine, E.D., 1994. Age differences in the personality characteristics of suicide completers: Preliminary findings from a psychological autopsy study. Psychiatry Interpers. Biol. Process. 57, 213-224.

Duberstein, P.R., Conwell, Y., Seidlitz, L., Denning, D.G., Cox, C., Caine, E.D., 2000. Personality traits and suicidal behavior and ideation in depressed inpatients 50 years of age and older. J. Gerontol. B. Psychol. Sci. Soc. Sci. 55, P18-26. https://doi.org/10.1093/geronb/55.1.p18 
Franklin, J.C., Ribeiro, J.D., Fox, K.R., Bentley, K.H., Kleiman, E.M., Huang, X., Musacchio, K.M., Jaroszewski, A.C., Chang, B.P., Nock, M.K., 2017. Risk factors for suicidal thoughts and behaviors: A meta-analysis of 50 years of research. Psychol. Bull. 143, 187-232. https://doi.org/10.1037/bul0000084

Harris, K., Gooding, P., Haddock, G., Peters, S., 2019. Factors that contribute to psychological resilience to suicidal thoughts and behaviours in people with schizophrenia diagnoses: qualitative study. BJPsych Open 5, 1-9. https://doi.org/10.1192/bjo.2019.63

Heisel, M.J., Neufeld, E., Flett, G.L., 2016. Reasons for living, meaning in life, and suicide ideation: investigating the roles of key positive psychological factors in reducing suicide risk in community-residing older adults. Aging Ment. Health 20, 195-207. https://doi.org/10.1080/13607863.2015.1078279

Hirsh, J., Deyoung, C., Peterson, J., 2009. Metatraits of the Big Five Differentially Predict Engagement and Restraint of Behavior. J. Pers. 77, 1085-102. https://doi.org/10.1111/j.1467-6494.2009.00575.x

Johnson, J.A., 2014. Measuring thirty facets of the five factor model with a 120-item public domain inventory: Development of the IPIP-NEO-120. J. Res. Personal. 51, 78-89. https://doi.org/10.1016/j.jrp.2014.05.003

Kaurin, A., Dombrovski, A.Y., Hallquist, M.N., Wright, A.G.C. Momentary interpersonal processes of suicidal surges in borderline personality disorder. Psychol. Med. 1-11. https://doi.org/10.1017/S0033291720004791

Kleiman, E.M., Nock, M.K., 2018. Real-time assessment of suicidal thoughts and behaviors. Curr. Opin. Psychol. 22, 33-37. https://doi.org/10.1016/j.copsyc.2017.07.026

Kleiman, E.M., Turner, B.J., Fedor, S., Beale, E.E., Huffman, J.C., Nock, M.K., 2017. Examination of real-time fluctuations in suicidal ideation and its risk factors: Results from two ecological momentary assessment studies. J. Abnorm. Psychol. 126, 726-738. https://doi.org/10.1037/abn0000273

Klibert, J.J., Luna, A., Miceli, M., 2019. "Savoring” buffers the association between negative emotions and suicidal behaviors in the GSM community. J. Gay Lesbian Ment. Health 23, 27-44. https://doi.org/10.1080/19359705.2018.1518795

Kovacs, M., Beck, A.T., 1977. The wish to die and the wish to live in attempted suicides. J. Clin. Psychol. 33, 361-365. https://doi.org/10.1002/1097-4679(197704)33:2<361::AIDJCLP2270330207>3.0.CO;2-H

Lee, Y., Oh, K.J., 2012. Validation of Reasons for Living and Their Relationship with Suicidal Ideation in Korean College Students. Death Stud. 36, 712-722. https://doi.org/10.1080/07481187.2011.584011

Linehan, M.M., Goodstein, J.L., Nielsen, S.L., Chiles, J.A., 1983. Reasons for staying alive when you are thinking of killing yourself: The Reasons for Living Inventory. J. Consult. Clin. Psychol. 51, 276-286. https://doi.org/10.1037//0022-006x.51.2.276

Linehan, M.M., Heard, H.L., Armstrong, H.E., 1993. Naturalistic follow-up of a behavioral treatment for chronically parasuicidal borderline patients. Arch. Gen. Psychiatry 50, $971-$ 974. https://doi.org/10.1001/archpsyc.1993.01820240055007

Linehan, M.M., Korslund, K.E., Harned, M.S., Gallop, R.J., Lungu, A., Neacsiu, A.D., McDavid, J., Comtois, K.A., Murray-Gregory, A.M., 2015. Dialectical behavior therapy for high suicide risk in individuals with borderline personality disorder: a randomized clinical trial and component analysis. JAMA Psychiatry 72, 475-482. https://doi.org/10.1001/jamapsychiatry.2014.3039 
Loranger, A.W., 1999. International Personality Disorder Examination: IPDE; DSM-IV and ICD-10; Interviews. PAR.

Malone, K.M., Haas, G.L., Sweeney, J.A., Mann, J.J., 1995. Major depression and the risk of attempted suicide. J. Affect. Disord. 34, 173-185. https://doi.org/10.1016/01650327(95)00015-f

McMakin, D.L., Siegle, G.J., Shirk, S.R., 2011. Positive Affect Stimulation and Sustainment (PASS) Module for Depressed Mood: A Preliminary Investigation of Treatment-Related Effects. Cogn. Ther. Res. 35, 217-226. https://doi.org/10.1007/s10608-010-9311-5

Muthén, L.K. and Muthén, B.O. (1998-2017). Mplus User's Guide. Eighth Edition. Los Angeles, CA: Muthén \& Muthén

O’Connor, S.S., Jobes, D.A., Yeargin, M.K., Fitzgerald, M.E., Rodríguez, V.M., Conrad, A.K., Lineberry, T.W., 2012. A cross-sectional investigation of the suicidal spectrum: typologies of suicidality based on ambivalence about living and dying. Compr. Psychiatry 53, 461-467. https://doi.org/10.1016/j.comppsych.2011.09.007

Oquendo, M.A., Dragatsi, D., Harkavy-Friedman, J., Dervic, K., Currier, D., Burke, A.K., Grunebaum, M.F., Mann, J.J., 2005. Protective Factors Against Suicidal Behavior in Latinos. J. Nerv. Ment. Dis. 193, 438-443. https://doi.org/10.1097/01.nmd.0000168262.06163.31

Pinto, A., Whisman, M.A., Conwell, Y., 1998. Reasons for living in a clinical sample of adolescents. J. Adolesc. 21, 397-405. https://doi.org/10.1006/jado.1998.0173

Posner, K., Brown, G.K., Stanley, B., Brent, D.A., Yershova, K.V., Oquendo, M.A., Currier, G.W., Melvin, G.A., Greenhill, L., Shen, S., Mann, J.J., 2011. The Columbia-Suicide Severity Rating Scale: initial validity and internal consistency findings from three multisite studies with adolescents and adults. Am. J. Psychiatry 168, 1266-1277. https://doi.org/10.1176/appi.ajp.2011.10111704

Restifo, E., Kashyap, S., Hooke, G.R., Page, A.C., 2015. Daily monitoring of temporal trajectories of suicidal ideation predict self-injury: A novel application of patient progress monitoring. Psychother. Res. J. Soc. Psychother. Res. 25, 705-713. https://doi.org/10.1080/10503307.2015.1006707

Richardson-Vejlgaard, R., Sher, L., Oquendo, M.A., Lizardi, D., Stanley, B., 2009. Objections to suicide among depressed patients with alcohol use disorders. J. Affect. Disord. 117, 197201. https://doi.org/10.1016/j.jad.2009.01.005

Sadikaj, G., Wright, A.G.C., Dunkley, D., Zuroff, D., Moskowitz, D.S., 2019. Multilevel Structural Equation Modeling for Intensive Longitudinal Data: A Practical Guide for Personality Researchers. https://doi.org/10.31234/osf.io/hwj9r

Segal, D.L., Marty, M.A., Meyer, W.J., Coolidge, F.L., 2012. Personality, suicidal ideation, and reasons for living among older adults. J. Gerontol. - Ser. B Psychol. Sci. Soc. Sci. 67 B, 159-166. https://doi.org/10.1093/geronb/gbr080

Shneidman, E.S., Farberow, N.L., 1957. Some comparisons between genuine and simulated suicide notes in terms of Mowrer's concepts of discomfort and relief. J. Gen. Psychol. 56, 251-256. https://doi.org/10.1080/00221309.1957.9920335

Soloff, P.H., Chiappetta, L., 2017. Suicidal behavior and psychosocial outcome in Borderline Personality Disorder at 8-year follow-up. J. Personal. Disord. 31, 774-789. https://doi.org/10.1521/pedi_2017_31_280 
Soloff, P.H., Fabio, A., Kelly, T.M., Malone, K.M., Mann, J.J., 2005. High-Lethality Status in Patients with Borderline Personality Disorder. J. Personal. Disord. 19, 386-399. https://doi.org/10.1521/pedi.2005.19.4.386

Szücs, A., Szanto, K., Aubry, J.-M., Dombrovski, A.Y., 2018. Personality and Suicidal Behavior in Old Age: A Systematic Literature Review. Front. Psychiatry 9. https://doi.org/10.3389/fpsyt.2018.00128

Szücs, A., Szanto, K., Wright, A.G.C., Dombrovski, A.Y., 2020. Personality of late- and earlyonset elderly suicide attempters. Int. J. Geriatr. Psychiatry 35, 384-395. https://doi.org/10.1002/gps.5254

Temes, C.M., Frankenburg, F.R., Fitzmaurice, G.M., Zanarini, M.C., 2019. Deaths by Suicide and Other Causes Among Patients With Borderline Personality Disorder and PersonalityDisordered Comparison Subjects Over 24 Years of Prospective Follow-Up. J. Clin. Psychiatry 80, 18m12436. https://doi.org/10.4088/JCP.18m12436

Tsoh, J., Chiu, H.F.K., Duberstein, P.R., Chan, S.S.M., Chi, I., Yip, P.S.F., Conwell, Y., 2005. Attempted suicide in elderly Chinese persons: a multi-group, controlled study. Am. J. Geriatr. Psychiatry Off. J. Am. Assoc. Geriatr. Psychiatry 13, 562-571. https://doi.org/10.1176/appi.ajgp.13.7.562

Useda, J.D., Duberstein, P.R., Conner, K.R., Beckman, A., Franus, N., Tu, X., Conwell, Y., 2007. Personality differences in attempted suicide versus suicide in adults 50 years of age or older. J. Consult. Clin. Psychol. 75, 126-133. https://doi.org/10.1037/0022006X.75.1.126

Useda, J.D., Duberstein, P.R., Conner, K.R., Conwell, Y., 2004. Personality and attempted suicide in depressed adults 50 years of age and older: A facet level analysis. Compr. Psychiatry 45, 353-361. https://doi.org/10.1016/j.comppsych.2004.06.002

Watson, D., Clark, L.A., Tellegen, A., 1988. Development and validation of brief measures of positive and negative affect: The PANAS scales. J. Pers. Soc. Psychol. 54, 1063-1070. https://doi.org/10.1037/0022-3514.54.6.1063

Witte, T.K., Fitzpatrick, K.K., Warren, K.L., Schatschneider, C., Schmidt, N.B., 2006. Naturalistic evaluation of suicidal ideation: Variability and relation to attempt status. Behav. Res. Ther. 44, 1029-1040. https://doi.org/10.1016/j.brat.2005.08.004 


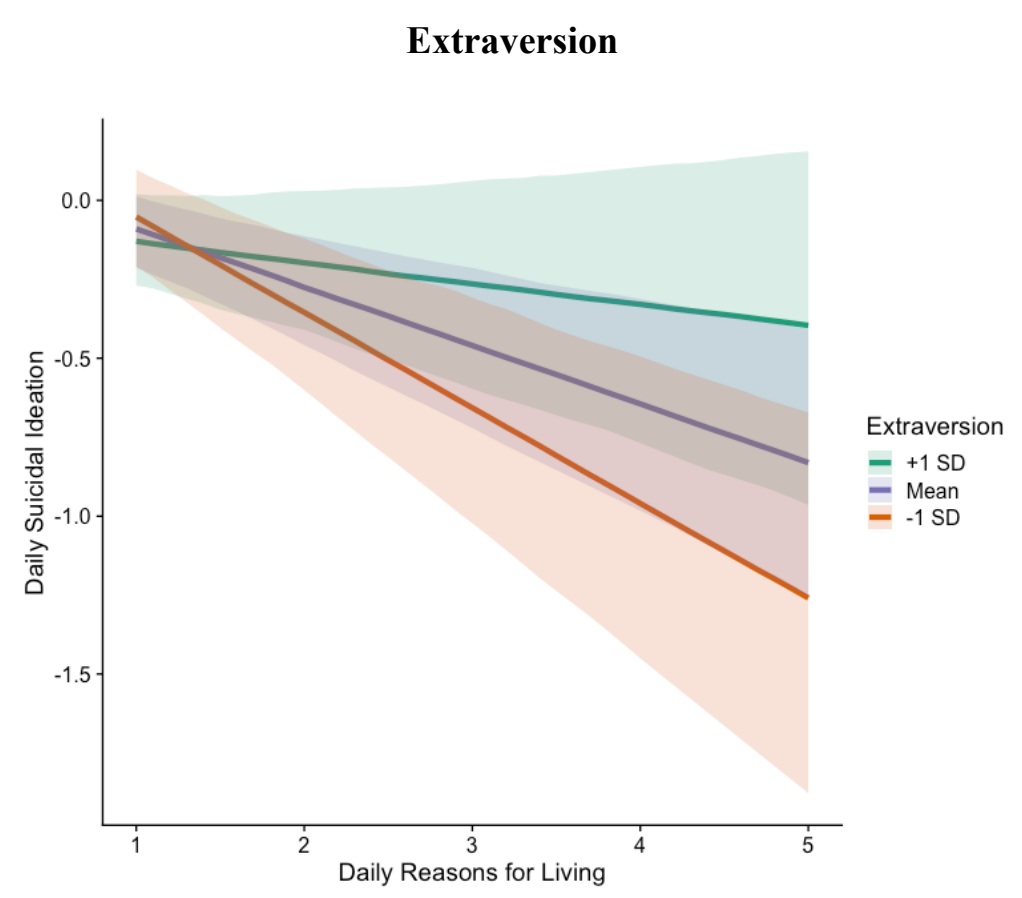

Figure 1. Distribution of random effects of RFL on suicidal ideation as a function of extraversion. Purple (middle) lines show the average expected effect at mean levels of extraversion, green (top) lines for +1 standard deviation (SD) above and orange (bottom) lines for -1 SD below the mean. 


\section{Table 1}

Descriptive Information for Key Study Variables

\begin{tabular}{|c|c|c|c|c|}
\hline & & $\begin{array}{c}\text { Total Sample } \\
\qquad N=153\end{array}$ & $\begin{array}{c}\text { BPD-NON } \\
n=48\end{array}$ & $\begin{array}{c}\text { ATT } \\
n=105\end{array}$ \\
\hline & $I C C$ & $M(S D)$ & $M(S D)$ & $M(S D)$ \\
\hline Age $M(S D)$ & & $33.69(9.60)$ & $32.25(6.78)$ & $34.34(10.61)$ \\
\hline Sex $(\%$ female $)$ & & 81.70 & 75.00 & 84.76 \\
\hline Suicidal ideation & & $0.31(0.72)$ & $0.14(0.39)$ & $0.39(0.82)$ \\
\hline \multicolumn{5}{|l|}{ Personality } \\
\hline Openness & & $89.32(12.49)$ & $91.82(10.79)$ & $88.19(13.07)$ \\
\hline Conscientiousness & & $74.48(15.12)$ & $76.11(14.24)$ & $73.75(15.52)$ \\
\hline Extraversion & & $69.86(17.24)$ & $70.07(15.03)$ & $69.77(18.22)$ \\
\hline Agreeableness & & $85.40(13.39)$ & $84.87(15.78)$ & $85.64(12.24)$ \\
\hline Neuroticism & & $89.22(13.85)$ & $90.51(14.14)$ & $88.64(13.75)$ \\
\hline \multicolumn{5}{|l|}{ Reasons for Living } \\
\hline Total & .63 & $2.41(0.79)$ & $2.57(0.70)$ & $2.33(0.82)$ \\
\hline Adjust/Cope with Problems & .49 & $2.29(0.84)$ & $2.50(0.76)$ & $2.20(0.87)$ \\
\hline Control over Life/Destiny & .56 & $2.19(0.94)$ & $2.44(0.90)$ & $2.08(0.94)$ \\
\hline Responsibilities to Family & .55 & $2.71(1.06)$ & $2.75(0.91)$ & $2.69(1.12)$ \\
\hline Love/Enjoy Family & .63 & $2.66(1.12)$ & $2.77(1.07)$ & $2.61(1.14)$ \\
\hline Future Plans & .49 & $2.49(0.96)$ & $2.69(0.92)$ & $2.40(0.96)$ \\
\hline Work Out for the Best & .51 & $2.11(0.85)$ & $2.29(0.85)$ & $2.03(0.84)$ \\
\hline
\end{tabular}

Note. BPD-NON: non-attempters diagnosed with BPD; ATT: suicide attempters diagnosed with BPD.

ICC: intraclass correlation. M: mean. SD: standard deviation. 


\section{Table 2}

Group-Wise Key Standardized Coefficients from Multilevel Structural Equation Models Predicting Suicidal Ideation from RFL

\begin{tabular}{|c|c|c|c|c|c|c|}
\hline & \multicolumn{2}{|c|}{ Total Sample } & \multicolumn{3}{|c|}{ BPD-NON } & \multirow{2}{*}{$\begin{array}{c}\text { ATT } \\
\text { CI }\end{array}$} \\
\hline & $\beta$ & $\mathrm{CI}$ & $\beta$ & $\mathrm{CI}$ & $\beta$ & \\
\hline \multicolumn{7}{|l|}{ within-person } \\
\hline $\mathrm{RFL} \rightarrow$ suicidal ideation & -.13 & $-.18 ;-.08$ & -.13 & $-.19 ;-.05$ & -.14 & $-.19 ;-.08$ \\
\hline \multicolumn{7}{|l|}{ between-person } \\
\hline $\mathrm{RFL} \rightarrow$ suicidal ideation & -.02 & $-.13 ; .13$ & -.09 & $-.30 ; .07$ & -.01 & $-.18 ; .20$ \\
\hline $\mathrm{SI} \rightarrow$ random slope & -.64 & $-.74 ;-.52$ & -.99 & $-.99 ;-.91$ & -.56 & $-.73 ;-.32$ \\
\hline
\end{tabular}


Table 3

Key Standardized Coefficients from Multilevel Structural Equation Models Predicting Suicidal Ideation from RFL and Prediction by Personality Traits

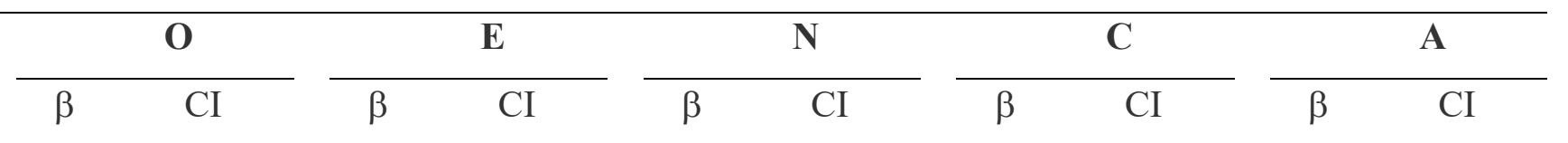

\begin{tabular}{|c|c|c|c|c|c|c|c|c|c|c|}
\hline \multicolumn{11}{|l|}{ within-person } \\
\hline RFL $\rightarrow$ suicidal ideation & -.12 & $-.18 ;-.07$ & -.13 & $-.18 ;-.08$ & -.12 & $-.17 ;-.07$ & -.12 & $-.18 ;-.07$ & -.12 & $-.18 ;-.07$ \\
\hline \multicolumn{11}{|l|}{ between-person } \\
\hline RFL $\rightarrow$ suicidal ideation & -.04 & $-.20 ; .12$ & -.02 & $-.18 ; .15$ & -.04 & $-.20 ; .11$ & -.06 & $-.23 ; .08$ & -.06 & $-.22 ; .09$ \\
\hline $\begin{array}{l}\text { Personality } \rightarrow \text { suicidal } \\
\text { ideation }\end{array}$ & -.11 & $-.29 ; .06$ & -.18 & $-.36 ; .02$ & .12 & $-.07 ; .26$ & .06 & $-.14 ; .22$ & -.02 & $-.20 ; .14$ \\
\hline Personality $\rightarrow \mathrm{RFL}$ & .20 & $.04 ; .40$ & .36 & $.19 ; .52$ & -.29 & $-.44 ;-.12$ & .10 & $-.07 ; .29$ & .01 & $-.16 ; .17$ \\
\hline Personality $\rightarrow$ random slope & .02 & $-.19 ; .20$ & .28 & $.06 ; .44$ & -.05 & $-.29 ; .18$ & .03 & $-.17 ; .23$ & .01 & $-.18 ; .18$ \\
\hline $\begin{array}{l}\text { Suicidal ideation } \rightarrow \text { random } \\
\text { slope }\end{array}$ & -.62 & $-.73 ;-.49$ & -.61 & $-.73 ;-.48$ & -.63 & $-.73 ;-.50$ & -.62 & $-.73 ;-.49$ & -.62 & $-.74 ;-.49$ \\
\hline
\end{tabular}

Note. $N=153$ (between), $N=2993$ (within); $\rightarrow$ indicates regression. Model parameter estimates are standardized. Bolded values indicate that $95 \%$ credibility interval of parameter estimates $(\mathrm{CI})$ does not contain zero. 